\title{
Treatment of stress fracture of the olecranon in throwing athletes with internal fixation through a small incision
}

\author{
Hiroyuki Fujioka ${ }^{1 *}$, Kenjiro Tsunemi ${ }^{2}$, Yohei Takagi ${ }^{2}$ and Juichi Tanaka ${ }^{2}$
}

\begin{abstract}
The present study is a report of retrospective case series of stress fracture of the olecranon. Six patients presented posterior elbow pain in throwing in baseball and softball, but fracture was not diagnosed in radiographs. We detected stress fracture of the olecranon using computed tomographic (CT) scan and treated the patient with internal fixation with a headless cannulated double threaded screw through a small skin incision. All patients returned to competitive level without elbow complaints after the operation.

When throwing athletes present with unusual posterior elbow pain and no significant findings on radiographs, a CT scan examination should be performed. We recommend surgical treatment of internal fixation with a screw through a small skin incision, as a good option for stress fracture of the olecranon in order to allow early return to sports activity in competitive athletes.
\end{abstract}

Keywords: Stress fracture, Elbow, Olecranon, Throwing athlete

\section{Background}

Overuse injuries of the elbow joint in throwing athletes, such as osteochondritis dissecans of the capitelum humeri at the lateral side of the elbow joint and insufficiency of the medial collateral ligament at the medial side of the elbow joint, are common in sports medicine. Osteoshondrosis of the capitelum humeri are caused by impaction of the humeroradial joint and injuries of the medial collateral ligament are caused by excessive traction force due to valgus stress applied to the elbow during throwing $[1,2]$.

By contrast, injuries to the posterior side of the elbow joint, which are less common, are known as stress fracture and epiphiseal injury of the olecranon and osetophyte formation of the olecranon. These injuries are caused by impingement of the olecraonon in the olecranon fossa of the humerus during valgus extension overload stress in pitching and by excessive pulling of the triceps brachii muscle on the olecranon during the acceleration phase of the throwing [3-6]. There are three

\footnotetext{
* Correspondence: hfujioka@huhs.ac.jp

'Department of Physical Therapy, Hyogo University of Health Sciences

School of Rehabilitation, 1-3-6 Minatojima, Chuo-ku, Kobe 650-8530, Japan

Full list of author information is available at the end of the article
}

types of fractures of the olecranon: the first type is a fracture of the tip of the olecranon osteophyte due to impingement of the olecranon fossa $[3,6]$; the second type is a fracture of the growth plate preventing the closure of the olecranon epiphysis seen in skeletally immature patients [4,5,7]; the third type is a straight or oblique fracture line in the middle third of the olecranon seen in the skeletally mature patients and this type of stress fracture of the olecranon caused by repetitive stress forces have been infrequently reported as a cause of elbow pain in adult throwing athletes [8-10].

In this report, we present the clinical features of a stress fracture of the olecranon in six skeletally mature patients who were treated with internal fixation through a small incision.

\section{Case presentation}

Six adolescent throwing athletes, 5 baseball pitchers and 1 softball pitcher, were included in this retrospective study (Table 1). All patients felt posterior elbow pain on throwing without any major causes. Although they suspended throwing for several weeks, the symptoms did not decrease and they visited us. Tenderness was detected at the posterior site of the olecranon, however, 
Table 1 Summary of the cases

\begin{tabular}{cccccc}
\hline Case & Age & Sex & Sport Played & $\begin{array}{c}\text { Duration From } \\
\text { Onset to Surgery }\end{array}$ & $\begin{array}{c}\text { Duration until Return to } \\
\text { Competition Level after Surgery }\end{array}$ \\
\hline 1 & 18 & M & Baseball Pitcher & 1 year and 4 months & 4 months \\
2 & 16 & M & Baseball Pitcher & 5 months & 6 months \\
3 & 17 & M & Baseball Pitcher & 7 months & 6 months \\
4 & 18 & F & Softball Pitcher & 6 months & 4 months \\
5 & 19 & M & Baseball Pitcher & 6 months & 6 months \\
6 & 21 & M & Baseball Pitcher & 2 weeks & 6 months \\
\hline
\end{tabular}

All patients were treated with internal fixation using DTJ large screw through a small incision and the implant was not removed.

range of motion of the elbow was normal. In all cases, radiographs of the elbow showed neither osteophyte formation nor osteosclerotic change, but computed tomographic $(\mathrm{CT})$ scan revealed a fracture line of the olecranon.

Although all patients reduced their sports activities for several weeks before visited us, elbow pain continued. Therefore we treated the patients surgically with internal fixation through small skin incision. The average duration from the onset to surgery was 6.8 months (range: 2 weeks to 1 year and 4 months). The average duration of follow-up was 2 years and 4 months (range: 2 to 3 years).

All patients were surgically treated with internal fixation with DTJ (Double Threaded screw Japan, Meira, Japan) large screw, which is a cannulated double threaded headless screw. Under general anesthesia, on the lateral decubitus position, a longitudinal 2 or $3 \mathrm{~cm}$ skin incision was made on the proximal site of the olecranon. A guide wire was fluoroscopically inserted to the ulna, from the lateral tip of the olecranon to the medial distal cortex of the ulna, crossing the fracture site. After drilling and tapping, an appropriate size screw was inserted through a small skin incision with neither exposure of the fracture site nor bone graft at the fracture site. The distal thread reached the medial cortex of the ulna and the proximal thread was located under the proximal end of the olecranon.

After two weeks immobilization with long arm cast, range of motion and muscle strengthening exercises, such as elbow extension and flexion exercise, forearm pronation and supination exercises, and wrist extension and flexion exercise, were started. Once full range of motion was obtained (4-6 weeks postoperatively) a gradual return to throwing program was started. At postoperative 4 months, the patients were allowed to return to competition level.

All patients had neither elbow pain during throwing nor loss of range of motion of the elbow and could return to competitive level, with almost the same performance level as before injury, at 6 months after surgery. The implant did not need to be removed in all patient.

\section{Representative case (case 2)}

The patient, a 16 year-old-man, was a high school baseball pitcher. He felt pain in the posterior aspects of the right elbow during throwing. Pain increased progressively until he was unable to throw. Physical examination revealed tenderness on the olecranon of the involved right elbow, however, there was no limitation of range of motion in the elbow. There were no significant signs such as fracture or osteoarthritic change on anteroposterior and lateral views of the radiographs (Figure 1A,B). However, CT scan clearly revealed a nondisplaced and straight oblique fracture line from the medial to the lateral at the middle third of the olecranon (Figure 1C,D). We diagnosed the patient with stress fracture of the olecranon and treated him with internal fixation using DTJ large screw as described above (Figure 2). The operated elbow was immobilized with long arm cast for two weeks after surgery. Thereafter, the patient started rehabilitation program of active range of motion and strengthening muscles. The patient returned to competitive baseball without elbow symptoms 6 months after the internal fixation.

\section{Discussion}

Stress fractures are partial or complete fractures of a bone resulting from an inability to withstand stress applied in a repeated manner [11]. Stress fractures of the lower extremities are common injuries in physically active athletes, however, stress fractures of the upper extremities are not common. In the upper extremities, stress fracture of the first rib, the olecranon, and the metacarpal bone have been reported as sports related injuries [11]. Because stress fracture is caused by overuse and fatigue of the surrounding musculature, most stress fractures heal with conservative treatment, such as rest and structured rehabilitation. In order to prevent throwing-related elbow pain in adolescent overhead athletes, improving rotator cuff strength with posterior shoulder muscle strengthening and scapular stabilization are critical parts of elbow rehabilitation $[12,13]$.

Nuber and Diment presented two cases of baseball players with non-displaced stress fractures detected as 

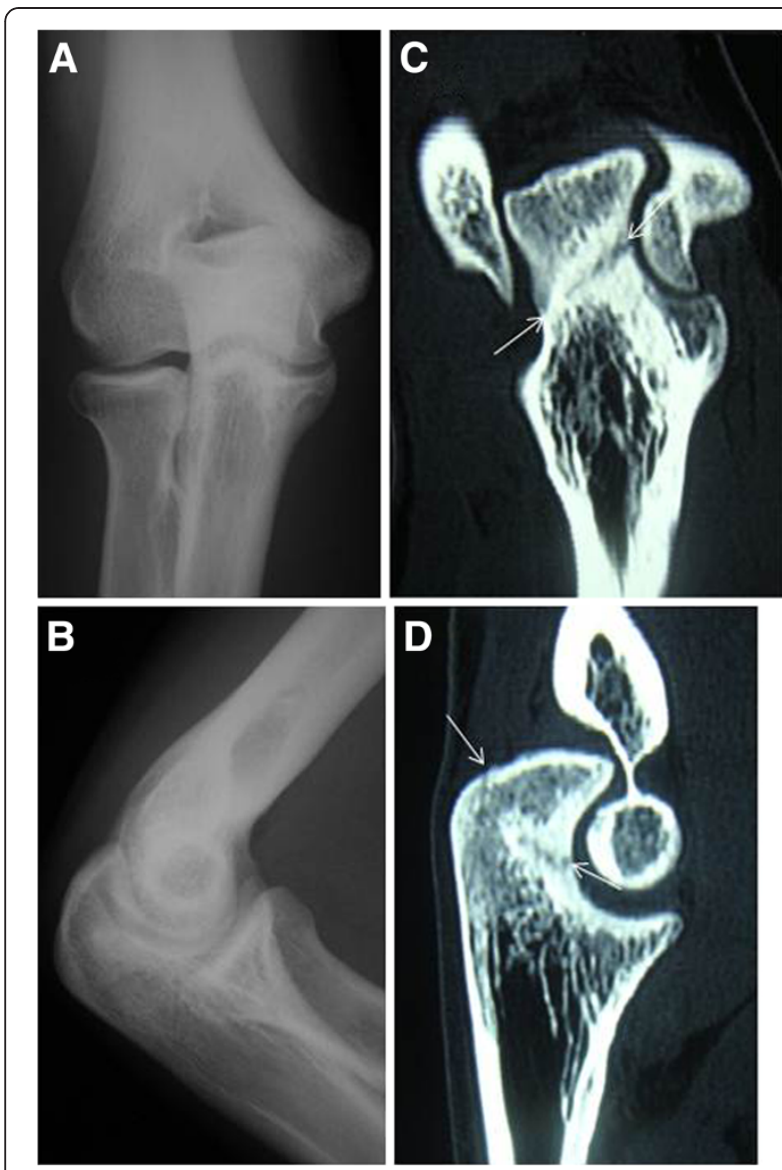

Figure 1 Case 2. Radiographs and CT scan of the elbow before surgery. There were no significant signs such as fracture or osteoarthritic change on anteroposterior $(\mathbf{A})$ and lateral $(\mathbf{B})$ views of radiographs. On the frontal $(\mathbf{C})$ and sagittal plane (D) of CT scan, non-displaced and straight oblique fracture line (arrow), from medial proximal to lateral distal at the middle third of the olecranon, was detected.

straight oblique fracture line at the middle third of the olecranon that were treated conservatively [10]. However they suggested that the long period of complete rest and immobilization for treatment was needed and that the fractures had the potential to displace due to traction force by the triceps brachii muscle.

Hulkko et al. reported that two javelin throwers at international level had oblique stress fractures at the middle third of the olecranon and were surgically treated with a tension band and two Kirschner wires [8]. These fractures healed in 4 months with no symptoms, however, one patient suffered a re-fracture and was treated surgically again. They recommend stress fractures of the olecranon be treated surgically in javelin throwers because of the high risk of delayed union. Nakaji et al. reported an olecranon stress fracture treated with open reduction and internal fixation with tension band wiring, however, the patient was suffering from re-fracture of
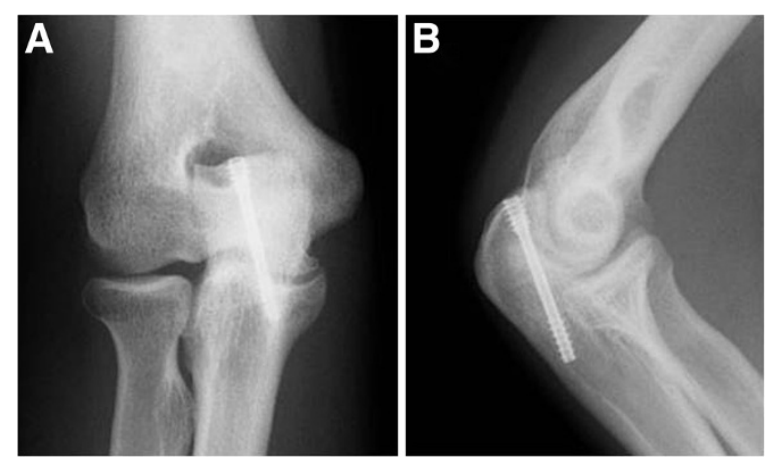

Figure 2 Case 2. Radiographs after surgery. The screw was inserted through a small incision with neither fracture site open nor bone graft and the fracture site was fixed with the screw on anteroposterior (A) and lateral (B) views of the radiographs. The distal thread of the screw reached the medial cortex of the ulna and the proximal thread was located in the cancellous bone of the olecranon.

the olecranon after implant removal and a revision surgery with internal fixation using a screw was performed [9]. They recommend surgical treatment of internal fixation with screw in competitive baseball players.

In order to detect stress fracture or occult fracture, CT scan, magnetic resonance imaging (MRI), and bone scintigraphy are all useful methods $[14,15]$. In athletes with early tibial stress injuries, multidetector CT scan has a good performance in the identification of cortical abnormality such as osteopenia, which is the earliest sign of fatigue damage of the cortical bone, and MRI has a good performance to evaluate soft tissue damage, perioseteal edema, and bone marrow edema [14]. In occult scaphoid fracture, multidetector CT scan is superior to dipect cortical involvement [15]. In our report, patients presented with pain at the posterior site of the elbow joint on throwing and fractures of the olecranon could not be detected on the radiographs but were visible on CT scans. Because stress fracture presents with gradual onset of pain in the elbow on throwing over a period of several weeks and the fracture line cannot usually be seen on radiographs, appropriate diagnosis may be delayed. CT scan is a useful examination to diagnose this problem.

In this report, all patients were treated with internal fixation with headless screw and the screws were not necessary to be removed in any patients. All patients had neither elbow pain during throwing nor loss of range of motion of the elbow and could return to competitive level.

\section{Conclusions}

When throwing athletes present with unusual posterior elbow pain and no significant findings on radiographs, a CT scan should be taken. In the competitive athletes 
required high performance that the fracture of the olecranon is detected on a CT scan, the fracture should be surgically treated with internal fixation with a screw through a small incision. Internal fixation using a headless double threaded cannulated screw was useful for dependable and early return to competitive level.

\section{Consent}

Written informed consent was obtained from all patients for publication of this case report and any accompanying images.

\section{Abbreviations}

CT: Computed tomography; MRI: Magnetic resonance imaging.

\section{Competing interests}

The authors delicate that they have no competing interests.

\section{Authors' contributions}

All authors contributed towards the treatment of the patients and in the manuscript preparation and approved the final manuscript.

\section{Author details}

${ }^{1}$ Department of Physical Therapy, Hyogo University of Health Sciences School of Rehabilitation, 1-3-6 Minatojima, Chuo-ku, Kobe 650-8530, Japan. ${ }^{2}$ Department of Orthopaedic Surgery, Hyogo College of Medicine, 1-1 Mukogawa-cho, Nishinomiya 663-8501, Japan.

Received: 18 January 2012 Accepted: 5 December 2012

Published: 14 December 2012

\section{References}

1. Lynch JR, Waitayawinyu T, Hanel DP, Trumble TE: Medial collateral ligament injury in the overhead throwing-athletes. J Hand Surg Am 2008, 33:430-437.

2. Yadao MA, Field LD, Savoie FH 3rd: Osteochondritis dissecans of the elbow. Instr Course Lect 2004, 53:599-606.

3. King JW, Brelsford HJ, Tullos HS: Analysis of the pitching arm of the professional baseball pitcher. Clinl Orthop Rel Res 1969, 67:116-123.

4. Rettig AC, Wurth TR, Mieling P: Nonunion of olecranon stress fractures in adolescent baseball pitchers. A case series of 5 athletes. Am J Sports Med 2006, 34:653-656.

5. Torg JS, Moyer RA: Non-union of a stress fracture through the olecranon epiphyseal plate observed in an adolescent baseball pitcher. A case report. J Bone Joint Surg Am 1977, 59:264-265.

6. Wilson FD, Andrews JR, Blackburn TA, McCluskey G: Valgus extension overload in the pitching elbow. Am J Sports Med 1983, 11:83-88

7. Charlton WP, Chandler RW: Persisitence of the olecranon physis in baseball players: Results following operative management. J Shoulder Elbow Surg 2003, 12:59-62.

8. Hulkko A, Orava S, Nikula P: Stress fractures of the olecranon in javelin throwers. Int J Sports Med 1986, 7:210-213.

9. Nakaji N, Fujioka H, Tanaka J, Sugimoto K, Yoshiya S, Fujita K, Kurosaka M: Stress fracture of the olecranon in an adult baseball player. Knee Surg Sports Traumatol Arthrosc 2006, 14:390-393.

10. Nuber GW, Diment MT: Olecranon stress fractures in throwers. A report of two cases and a review of the literature. Clinl Orthop Rel Res 1992, 278:58-61.

11. Jones GL: Upper extremity stress fractures. Clin Sports Med 2006, 25:159-174.

12. Ellenbecker TS, Reinold M, Nelson CO: Clinical concepts for treatment of the elbow in the adolescent overhead athlete. Clin Sports Med 2010, 29:705-724.

13. Trakis JE, McHugh MP, Caracciolo PA, Buscicco L, Mullaney M, Nicholas SJ: Muscle strength and range of motion in adolescent pitchers with throwing-related pain: implications for injury prevention. Am J Sports Med 2008, 36:2173-2178.

14. Gaeta M, Minutoli F, Scribano E, Ascenti G, Vinci S, Bruschetta D, Magaudda $L$, Blandino $A: C T$ and $M R$ imaging findings in athletes with early tibial stress injuries: comparison with bone scintigraphy finsings and emphasis on cortical abnormalities. Radiology 2005, 235:553-561.

15. Memarsadeghi M, Breitenseher MJ, Schaefer-Prokop C, Weber M, Aldrian S, Gäbler C, Prokop M: Occult scaphoid fractures: comparison of multidetector CT and MR imaging-Initial experience. Radiology 2006, 240:169-176.

doi:10.1186/1758-2555-4-49

Cite this article as: Fujioka et al:: Treatment of stress fracture of the olecranon in throwing athletes with internal fixation through a small incision. Sports Medicine, Arthroscopy, Rehabilitation, Therapy \& Technology 2012 4:49.

\section{Submit your next manuscript to BioMed Central and take full advantage of:}

- Convenient online submission

- Thorough peer review

- No space constraints or color figure charges

- Immediate publication on acceptance

- Inclusion in PubMed, CAS, Scopus and Google Scholar

- Research which is freely available for redistribution 